\title{
ON A FREQUENCY LOCALIZED BERNSTEIN INEQUALITY AND SOME GENERALIZED POINCARÉ-TYPE INEQUALITIES
}

\author{
DONG LI
}

\begin{abstract}
We consider a frequency localized Bernstein inequality for the fractional Laplacian operator, which has wide applications in fluid dynamics such as dissipative surface quasi-geostrophic equations. We use a heat flow reformulation and prove the inequality for the full range of parameters and in all dimensions. A crucial observation is that after frequency projection the zeroth frequency part of the Lévy semigroup does not participate in the inequality and therefore can be freely adjusted. Our proof is based on this idea and a careful perturbation of the Lévy semigroup near the zero frequency, which preserves the positivity and improves the time decay. As an application we also give new proofs of some generalized Poincaré-type inequalities.
\end{abstract}

\section{Introduction}

In this paper, we consider a frequency localized Bernstein-type inequality, which has useful applications in fluid dynamics. Let $\alpha>0$ and consider the fractional Laplacian operator $|\nabla|^{\alpha}$ defined via Fourier transform by the relation

$$
\widehat{|\nabla|^{\alpha} f}(\xi)=(2 \pi|\xi|)^{\alpha} \hat{f}(\xi), \quad \xi \in \mathbb{R}^{d} .
$$

Here $\hat{f}(\xi)$ is the usual Fourier transform of a scalar-valued function $f$ on $\mathbb{R}^{d}$. To fix the notations, we adopt the following conventional definition of Fourier transform pair:

$$
\begin{aligned}
\text { Fourier transform: } & (\mathcal{F} f)(\xi)=\hat{f}(\xi)=\int_{\mathbb{R}^{d}} f(x) e^{-2 \pi i x \cdot \xi} d x, \\
\text { Inverse Fourier transform: } & f(x)=\int_{\mathbb{R}^{d}} \hat{f}(\xi) e^{2 \pi i x \cdot \xi} d \xi .
\end{aligned}
$$

Occasionally, we also use the notation $\mathcal{F}^{-1}$ to denote inverse Fourier transform. Let $0<\alpha \leq 2$ and $1<q<\infty$, the Bernstein-type inequality we are interested in takes the following form: for any $A_{2}>A_{1}>0$ and any $f \in L^{q}\left(\mathbb{R}^{d}\right)$ with

$$
\operatorname{supp}(\hat{f}) \subset\left\{\xi: A_{1} \leq|\xi| \leq A_{2}\right\}
$$

there is a constant $C$ depending only on $\left(d, p, \alpha, A_{1}, A_{2}\right)$ such that

$$
C\|f\|_{q}^{q} \geq \int_{\mathbb{R}^{d}}\left(|\nabla|^{\alpha} f\right)|f|^{q-2} f d x \geq \frac{1}{C}\|f\|_{q}^{q} .
$$

Here $\|f\|_{q}$ is the usual Lebesgue norm of $f$ on $\mathbb{R}^{d}$. An equivalent and more commonly used formulation of (1.2) is stated in Corollary (1.6) in which the frequency support condition (1.1) is replaced by the Littlewood-Paley operators. In (1.2), the upper bound is trivial: it is a consequence of the Hölder's inequality and the usual Bernstein

Received by the editors December 22012. 
inequality. As for the lower bound, the case $q=2$ is a simple consequence of the Plancherel theorem since by (1.1),

$$
\begin{aligned}
\int_{\mathbb{R}^{d}}|\nabla|^{\alpha} f f d x & =\int_{A_{1} \leq|\xi| \leq A_{2}}(2 \pi|\xi|)^{\alpha}|\hat{f}(\xi)|^{2} d \xi \\
& \geq\left(2 \pi A_{1}\right)^{\alpha} \int_{\mathbb{R}^{d}}|\hat{f}(\xi)|^{2} d x=\left(2 \pi A_{1}\right)^{\alpha}\|f\|_{2}^{2} .
\end{aligned}
$$

It is the case $1<q<\infty, q \neq 2$ which requires more elaborate analysis.

For the full Laplacian case $\alpha=2$, the inequality (1.2) can be reduced to the form (still under the condition (1.1))

$$
\int_{\mathbb{R}^{d}}|\nabla f|^{2}|f|^{q-2} d x \geq C\|f\|_{q}^{q}
$$

after an integration by parts argument. The inequality (1.3) was first proved by Danchin [5] when $q$ is an even integer and under a certain $q$-dependent small angle condition on the frequency support. Planchon [11] proved the case $\alpha=2,2<q<\infty$ by using an integration by parts argument. In [6], Danchin settled the remaining case $\alpha=2,1<q<2$ in the appendix of that paper (see Lemma A.5 therein and the erratum in [7]). The fractional Laplacian formulation of (1.2) for $0<\alpha<2$ first appeared in $\mathrm{Wu}[12]$ and it is of fundamental importance in the wellposedness theory for the dissipative quasi-geostrophic equations. In [4], Chen et al. proved the inequality (1.2) for $0<\alpha<2,2<q<\infty$ by using an interpolation definition of Besov spaces. Recently Hmidi [9] even generalized (1.2) to some logarithm-damped fractional Laplacian operators of the form

$$
\frac{|\nabla|^{\gamma}}{\log ^{\beta}(\lambda+|\nabla|)}, \quad 0 \leq \beta \leq \frac{1}{2}, \quad 0 \leq \gamma \leq 1, \lambda \geq e^{\frac{3+2 \alpha}{\beta}}
$$

in dimensions $d=1,2,3$. The maximum principle for these nonlocal operators is obtained in [9] and [8].

The purpose of this note is to give a completely new proof of (1.2), which works for $0<\alpha<2$ and for all $1<q<\infty$. We begin by reformulating (1.2) in terms of a (fractional) heat flow estimate. The following result is the key step.

Theorem 1.1 (Improved heat flow estimate). Let the dimension $d \geq 1$. Let $0<\alpha<2$ and $1 \leq q \leq \infty$. There exists a constant $c>0$ depending only on the dimension $d$ and $\alpha$ such that for any $N>0$ and any function $f \in L_{x}^{q}\left(\mathbb{R}^{d}\right)$, we have

$$
\left\|e^{-t|\nabla|^{\alpha}} P_{N} f\right\|_{q} \leq e^{-c t N^{\alpha}}\left\|P_{N} f\right\|_{q}, \quad \forall t \geq 0 .
$$

Here $P_{N}$ is the Littlewood-Paley operator defined in (1.19). For $\alpha=2$, there is an absolute constant $\tilde{c}>0$ such that for any $1<q<\infty, f \in L_{x}^{q}\left(\mathbb{R}^{d}\right)$, we have

$$
\left\|e^{t \Delta} P_{N} f\right\|_{q} \leq e^{-\tilde{c} \frac{q-1}{q^{2}} t N^{2}}\left\|P_{N} f\right\|_{q}, \quad \forall t \geq 0 .
$$

Remark 1.2. The usual Young's inequality together with the fact $\left\|\mathcal{F}^{-1}\left(e^{-t(2 \pi|\xi|)^{\alpha}}\right)\right\|_{L_{x}^{1}}=$ 1 easily yields that

$$
\left\|e^{-t|\nabla|^{\alpha}} P_{N} f\right\|_{q} \leq\left\|P_{N} f\right\|_{q}, \quad \forall t \geq 0
$$


The inequalities (1.4) and (1.5) give a strengthening (thus the name "improved") of the above estimate. In [3] (see Lemma 2.1 therein), Chemin proved the estimate

$$
\left\|e^{-t|\nabla|^{\alpha}} P_{N} f\right\|_{q} \leq C_{1} e^{-c t N^{\alpha}}\left\|P_{N} f\right\|_{q}, \quad \forall t \geq 0
$$

with a non-sharp constant $C_{1}$. The inequality (1.6) is already good enough for most Partial Differential Equation (PDE) applications. However, the main point of (1.4) is that $C_{1}$ can take the sharp value 1 . This is very important for (and in fact the key to) deriving the later inequality (1.2). We shall only need the estimate near $t=0$ to prove the inequality (1.2). Also it is worthwhile pointing it out that in (1.4) $P_{N}$ can be replaced by $P_{\geq N}$ or $P_{>N}$ since the main property needed in the proof is a certain spectral gap condition.

Remark 1.3. We stress that the two bounds (1.4) and (1.5) are essentially optimal. In particular, the constant $c$ in (1.4) cannot be taken to be uniform for all $0<\alpha \leq 2$ and will actually blow up at $\alpha=2$. This is deeply connected with the fact that the spatial decay of $e^{-t|\nabla|^{\alpha}}$ is power-like only for $0<\alpha<2$. For the full Laplacian, even with frequency localization, one should not expect the inequality

$$
\left\|e^{t \Delta} P_{N} f\right\|_{\infty} \leq e^{-c t N^{2}}\left\|P_{N} f\right\|_{\infty}
$$

To see this point it suffices to consider the periodic case, see Remark 1.11 below.

Remark 1.4. By formally interpolating the obvious inequalities (here $0<\alpha \leq 2$, $N>0$, and $c_{1}$ is an absolute constant):

$$
\begin{aligned}
& \left\|e^{-t|\nabla|^{\alpha}} P_{N} f\right\|_{2} \leq e^{-c_{1} t N^{\alpha}}\left\|P_{N} f\right\|_{2}, \\
& \left\|e^{-t|\nabla|^{\alpha}} P_{N} f\right\|_{q} \leq\left\|P_{N} f\right\|_{q}, \quad q=1 \text { or } \infty,
\end{aligned}
$$

one is led to

$$
\left\|e^{-t|\nabla|^{\alpha}} P_{N} f\right\|_{q} \leq e^{-c_{1} t N^{\alpha} \cdot \frac{q-1}{q^{2}}}\left\|P_{N} f\right\|_{q}, \quad \forall 1<q<\infty .
$$

Comparing (1.8) with (1.4), the main improvement there is at the endpoints $q=1$ and $q=\infty$ for $0<\alpha<2$. In a recent paper [2] (see Theorem 5.3 therein), Chamorro and Lemarié proved the inequality

$$
\left\|e^{-t|\nabla|^{\alpha}} P_{N} f\right\|_{q} \leq e^{-c_{\alpha, q} t N^{\alpha}}\left\|P_{N} f\right\|_{q}, \quad \forall 1<q<\infty,
$$

with an implicit constant $c_{\alpha, q}>0$. Interestingly, their analysis proceeds by first establishing an infinitesimal inequality of the form

$$
q \int_{\mathbb{R}^{d}} P_{N} f\left|P_{N} f\right|^{q-2}(-\Delta) P_{N} f d x \geq c_{q} N^{2}\left\|P_{N} f\right\|_{q}^{q}, \quad 1<q<\infty,
$$

where $c_{q}>0$ depends on $q$ and the dimension $d$. Then they use the above inequality (1.10) to deduce (1.9).

Remark 1.5. One may wonder whether it is possible to absorb the frequency localization into the kernel and prove directly the bound (say for $N=1$ )

$$
\left\|\mathcal{F}^{-1}\left(P_{1} e^{-t|\nabla|^{\alpha}}\right)\right\|_{L_{x}^{1}} \leq e^{-c t}, \quad t>0
$$


We show that (1.11) is impossible even for $t$ sufficiently small. Let $\phi_{1}(\xi)=\varphi(\xi)-\varphi(2 \xi)$ (see (1.19) for the definition of $\varphi$ ) and consider the function

$$
g(t, x)=\int_{\mathbb{R}^{d}} e^{-t(2 \pi|\xi|)^{\alpha}} \phi_{1}(\xi) e^{2 \pi i \xi \cdot x} d \xi .
$$

For $|\xi|=1$, we have by definition

$$
\begin{aligned}
1=\left|\phi_{1}(\xi)\right| & =\left|\int_{\mathbb{R}^{d}} g(0, x) e^{-2 \pi i x \cdot \xi} d \xi\right| \\
& \leq \int_{\mathbb{R}^{d}}|g(0, x)||\cos (2 \pi x \cdot \xi)| d x \\
& \leq \int_{\mathbb{R}^{d}}|g(0, x)| d x .
\end{aligned}
$$

By examining the conditions for equality, it is not difficult to disprove the possibility $\|g(0, \cdot)\|_{1}=1$. Therefore, we have $\|g(0, \cdot)\|_{1}>1$. Since $\|g(t, \cdot)\|_{1}$ is continuous in $t$, we get $\|g(t, \cdot)\|_{1}>1$ for $t$ sufficiently small. This disproves (1.11).

The Bernstein inequality (1.2) can be regarded as an infinitesimal version of the decay estimate (1.4) and (1.5). We state it as the following corollary.

Corollary 1.6. Let the dimension $d \geq 1$. Let $0<\alpha<2$ and $1<q<\infty$. Then for any $N>0$, any $f \in L_{x}^{q}\left(\mathbb{R}^{d}\right)$,

$$
\int_{\mathbb{R}^{d}}\left(|\nabla|^{\alpha} P_{N} f\right)\left|P_{N} f\right|^{q-2} P_{N} f d x \geq c N^{\alpha}\left\|P_{N} f\right\|_{q}^{q},
$$

where the constant $c$ depends only on the dimension $d$ and $\alpha$. For $\alpha=2$, there is an absolute constant $\tilde{c}>0$ such that for any $1<q<\infty$, any $N>0$ and any $f \in L_{x}^{q}\left(\mathbb{R}^{d}\right)$, we have the inequality

$$
-\int_{\mathbb{R}^{d}}\left(\Delta P_{N} f\right)\left|P_{N} f\right|^{q-2} P_{N} f d x \geq \tilde{c} \frac{q-1}{q^{2}} N^{2}\left\|P_{N} f\right\|_{q}^{q} .
$$

Remark 1.7. In (1.12) $P_{N}$ can be replaced by $P_{\geq N}$ or $P_{>N}$ or other similar frequency projection operators. Note that in Corollary 1.6 the restriction of $q$ is $1<q<\infty$. This is because we shall deduce (1.12) and (1.13) from (1.4) and (1.5) through a differentiation argument. A rigorous justification of differentiating under the integral requires $1<q<\infty$.

Remark 1.8. We stress that for $0<\alpha<2$ the constant $c$ in (1.12) depends only on $(d, \alpha)$. In particular, it does not depend on the constant $q$. This is in sharp contrast with the full Laplacian case where the constant is proportional to $(q-1) / q^{2}$ which vanishes at $q=1$ and $q=\infty$. In [6] (see equation (88) on page 1228 therein), Danchin effectively proved the inequality $(1.13)$ by using an integration by parts argument. It should be possible to show that (1.12) and (1.13) are essentially optimal. But we will not dwell on this issue here.

Before we move on to other similar results, let us explain the "mechanism" of our proof. In some sense our proof is an upgraded version of the formal argument in Remark 1.4. In particular (1.4) is a strengthened version of (1.7) in the case $0<\alpha<2$ (and hence the improvement). The proof of (1.4) is based on frequency localization 
and Young's inequality. We briefly explain the idea as follows. First note that by scaling it suffices to prove (1.4) for $N=1$. By frequency localization we have

$$
\begin{aligned}
e^{-t(2 \pi|\xi|)^{\alpha}} \widehat{P_{1} f}(\xi) & =e^{-t(2 \pi|\xi|)^{\alpha}} \psi(\xi) \hat{f}(\xi) \\
& =e^{-t\left((2 \pi|\xi|)^{\alpha}+\epsilon \phi_{1}(\xi)\right)} \psi(\xi) \hat{f}(\xi),
\end{aligned}
$$

where $\psi(\xi)=\varphi(\xi)-\varphi(2 \xi)$ (see (1.19)) and $\phi_{1}(\xi)=\varphi(6 \xi)$. In the last equality, we used the fact that $\psi(\xi)=0$ for $|\xi| \leq 1 / 2$ and $\phi_{1}(\xi)=0$ for $|\xi| \geq 1 / 3$. Now to prove (1.4) it suffices to show that the modified kernel

$$
k_{\epsilon}(t, x)=\mathcal{F}^{-1}\left(e^{-t\left((2 \pi|\xi|)^{\alpha}+\epsilon \phi_{1}(\xi)\right)}\right)
$$

has the bound $\left\|k_{\epsilon}(t, \cdot)\right\|_{L_{x}^{1}} \leq e^{-c t}$ for some $c>0$. Since $\hat{k}_{\epsilon}(t, 0)=e^{-t \epsilon \phi_{1}(0)}=e^{-t \epsilon}$, we only need to prove that $k_{\epsilon}(t, x)$ is non-negative for $\epsilon$ sufficiently small. The proof of this fact is given in Lemma 2.1. The main idea is to use the slow (power-like) decay (see (2.9)) of the Lévy semigroup when $0<\alpha<2$ which is stable under smooth perturbations.

It is fairly interesting to establish some analogues of Theorem 1.1 and Corollary 1.6 in the periodic domain case. To fix notations, let the dimension $d \geq 1$ and $\mathbb{T}^{d}=\mathbb{R}^{d} / \mathbb{Z}^{d}$ be the usual periodic torus. For a smooth periodic function $f: \mathbb{T}^{d} \rightarrow \mathbb{R}$, we adopt the Fourier expansion and inversion formulae:

$$
\begin{aligned}
& f(x)=\sum_{k \in \mathbb{Z}^{d}} \hat{f}(k) e^{2 \pi i k \cdot x}, \quad x \in \mathbb{T}^{d}, \\
& \hat{f}(n)=\int_{\mathbb{T}^{d}} f(x) e^{-2 \pi i n \cdot x} d x, \quad n \in \mathbb{Z}^{d} .
\end{aligned}
$$

For $0<\alpha \leq 2$, the fractional Laplacian operator $|\nabla|^{\alpha}$ is defined by the relation

$$
\widehat{|\nabla|^{\alpha} f}(n)=(2 \pi|n|)^{\alpha} \hat{f}(n), \quad n \in \mathbb{Z}^{d} .
$$

We shall say a function $f \in L^{1}\left(\mathbb{T}^{d}\right)$ has mean zero if $\hat{f}(0)=0$ or equivalently

$$
\int_{\mathbb{T}^{d}} f(x) d x=0 .
$$

With these notations, we have

Theorem 1.9 (Improved heat flow estimate, periodic case). Let the dimension $d \geq 1$. For any $0<\alpha<2$, there is a constant $c_{1}>0$ depending only on $(\alpha, d)$ such that for any $1 \leq q \leq \infty$ and any $f \in L^{q}\left(\mathbb{T}^{d}\right)$ with mean zero, we have

$$
\left\|e^{-t|\nabla|^{\alpha}} f\right\|_{q} \leq e^{-c_{1} t}\|f\|_{q}, \quad \forall t>0 .
$$

For $\alpha=2$, there is an absolute constant $c_{2}>0$ such that for any $1<q<\infty$, $f \in L^{q}\left(\mathbb{T}^{d}\right)$ with mean zero, we have

$$
\left\|e^{t \Delta} f\right\|_{q} \leq e^{-c_{2} \frac{q-1}{q^{2}} t}\|f\|_{q}, \quad \forall t>0 .
$$

Remark 1.10. One should note again the subtle difference between the bounds (1.14) for $0<\alpha<2$ and (1.15) for $\alpha=2$. The main reason is that in the periodic setting our perturbation argument also relies heavily on the pointwise lower bound of the Lévy semigroup for sufficiently small $t$. When $0<\alpha<2$ the decay of $e^{-t|\nabla|^{\alpha}}$ is power-like. However, for $\alpha=2$, this is no longer the case and the perturbation argument does not work. 
Remark 1.11. We stress that (1.15) is optimal. In particular, one should not expect the inequality

$$
\left\|e^{t \Delta} f\right\|_{\infty} \leq e^{-c t}\|f\|_{\infty}
$$

even for $t>0$ sufficiently small. To see this, we take any smooth $f$ on $\mathbb{T}^{d}$ with $\|f\|_{\infty}=1$ and zero mean. Suppose $x_{0} \in \mathbb{T}^{d}$ and $f(x) \equiv 1$ in some neighborhood $\left|x-x_{0}\right| \leq \delta_{0}$ with $\delta_{0}>0$. Write

$$
e^{t \Delta} f=k(t, \cdot) * f
$$

where $*$ denote the usual convolution on $\mathbb{T}^{d}$ and $k(t, \cdot)$ is the periodic heat kernel. By using the Poisson summation formula (see Lemma 2.3), it is not difficult to check that for $|y| \leq \frac{1}{2}, 0<t<1$, we have

$$
k(t, y)=\frac{1}{(4 \pi t)^{\frac{d}{2}}} e^{-\frac{|y|^{2}}{4 t}}+O\left(e^{-\frac{C}{t}}\right),
$$

where $C>0$ is some constant. We then have for $t>0$ sufficiently small and some constant $C_{0}>0$,

$$
\begin{aligned}
\left|\left(e^{t \Delta} f\right)\left(x_{0}\right)\right| & \geq \int_{|y| \leq \frac{\delta_{0}}{2}} \frac{1}{(4 \pi t)^{\frac{d}{2}}} e^{-\frac{|y|^{2}}{4 t}} d y-O\left(e^{-\frac{C}{t}}\right) \\
& \geq 1-e^{-\frac{\delta_{0}^{2}}{64 t}} \int_{|y|>\frac{\delta_{0}}{2}} \frac{1}{(4 \pi t)^{\frac{d}{2}}} e^{-\frac{|y|^{2}}{8 t}} d y-O\left(e^{-\frac{C}{t}}\right) \\
& \geq 1-O\left(e^{-\frac{C_{0}}{t}}\right) .
\end{aligned}
$$

This disproves (1.16).

An immediate consequence of Theorem 1.9 is a family of generalized Poincaré-type inequalities.

Corollary 1.12 (Generalized Poincaré-type inequalities for periodic domains). Let the dimension $d \geq 1$. For any $0<\alpha<2$, there is a constant $c_{1}>0$ depending only on $(\alpha, d)$ such that for any any $f \in C^{2}\left(\mathbb{T}^{d}\right)$ with mean zero, we have

$$
\int_{\mathbb{T}^{d}}\left(|\nabla|^{\alpha} f\right)|f|^{q-2} f d x \geq c_{1}\|f\|_{q}^{q}, \quad \forall 1<q<\infty .
$$

For $\alpha=2$, there is an absolute constant $c_{2}>0$ such that for any $f \in C^{2}\left(\mathbb{T}^{d}\right)$ with mean zero, we have

$$
-\int_{\mathbb{T}^{d}}(\Delta f)|f|^{q-2} f d x \geq c_{2} \frac{q-1}{q^{2}}\|f\|_{q}^{q}, \quad \forall 1<q<\infty .
$$

The proof of Corollary 1.12 is quite similar to the proof of Corollary 1.6 and therefore we omit it.

Remark 1.13. In [10] (see Proposition A.14.1 on page 291 therein), by using a contradiction argument, the authors proved the inequality (1.18) for the case $2 \leq$ $q<\infty$ with a dimension-dependent constant. Our result here covers the whole range $1<q<\infty$ and $0<\alpha \leq 2$. 
We conclude the introduction by setting up some

Notations. We will need to use the Littlewood-Paley frequency projection operators. Let $\varphi(\xi)$ be a smooth bump function supported in the ball $|\xi| \leq 2$ and equal to one on the ball $|\xi| \leq 1$.

For two real positive numbers $\alpha<\beta$, define the frequency localized (LP) projection operator $P_{\alpha<\cdot<\beta}$ by

$$
P_{\alpha<<\beta} f=\mathcal{F}^{-1}\left(\left[\phi\left(\beta^{-1} \xi\right)-\phi\left(\alpha^{-1} \xi\right)\right] \mathcal{F}(f)\right)
$$

For any $N>0$, we denote by

$$
P_{N}:=P_{N / 2<\cdot<N},
$$

which projects the frequency to $|\xi| \sim N$.

Here $\mathcal{F}$ and $\mathcal{F}^{-1}$ denote the Fourier transform and its inverse transform, respectively. Similarly, the operators $P_{<\alpha}$ and $P_{>\beta}$ are defined by

$$
P_{<\beta} f=\mathcal{F}^{-1}\left(\phi\left(\beta^{-1} \xi\right) \mathcal{F}(f)\right)
$$

and

$$
P_{>\alpha} f=\mathcal{F}^{-1}\left(\left[1-\phi\left(\alpha^{-1} \xi\right)\right] \mathcal{F}(f)\right) .
$$

\section{Proof of main theorems}

We begin with a simple lemma. Let $\phi_{1} \in C_{c}^{\infty}\left(\mathbb{R}^{d}\right)$ be a radial function such that

$$
\phi_{1}(x)= \begin{cases}1, & |x| \leq \frac{1}{4} \\ 0, & |x| \geq \frac{1}{3}\end{cases}
$$

Let $\epsilon>0$ and define for $t>0$,

$$
F_{\epsilon}(t, x)=\int_{\mathbb{R}^{d}} e^{-t(2 \pi|\xi|)^{\alpha}}\left(e^{-\epsilon t \phi_{1}(\xi)}-1\right) e^{2 \pi i \xi \cdot x} d \xi .
$$

Also denote

$$
p(t, x)=\mathcal{F}^{-1}\left(e^{-t(2 \pi|\xi|)^{\alpha}}\right)=\int_{\mathbb{R}^{d}} e^{-t(2 \pi|\xi|)^{\alpha}} e^{2 \pi i \xi \cdot x} d \xi
$$

and

$$
k_{\epsilon}(t, x)=\mathcal{F}^{-1}\left(e^{-t\left((2 \pi|\xi|)^{\alpha}+\epsilon \phi_{1}(\xi)\right.}\right)=\int_{\mathbb{R}^{d}} e^{-t\left((2 \pi|\xi|)^{\alpha}+\epsilon \phi_{1}(\xi)\right)} e^{2 \pi i \xi \cdot x} d \xi
$$

Clearly

$$
k_{\epsilon}(t, x)=p(t, x)+F_{\epsilon}(t, x) .
$$

The following lemma shows that for $\epsilon>0$ sufficiently small $k_{\epsilon}(t, x)$ is still a positive kernel.

Lemma 2.1. Let $d \geq 1$ and $0<\alpha<2$. There exists a constant $C_{1}=C_{1}(d, \alpha)>0$ such that for any $x \in \mathbb{R}^{d}, t>0$,

$$
\frac{1}{C_{1}} \cdot \frac{t}{\left(t^{\frac{1}{\alpha}}+|x|\right)^{d+\alpha}} \leq p(t, x) \leq C_{1} \cdot \frac{t}{\left(t^{\frac{1}{\alpha}}+|x|\right)^{d+\alpha}} .
$$

There exists a constant $\epsilon_{0}=\epsilon_{0}(d, \alpha)>0$ such that if $0<\epsilon<\epsilon_{0}$, then

$$
p(t, x)-\left|F_{\epsilon}(t, x)\right|>0, \quad \forall x \in \mathbb{R}^{d}, 0<t \leq 1 .
$$


In particular,

$$
\left\|k_{\epsilon}(t, \cdot)\right\|_{L_{x}^{1}} \leq e^{-\epsilon t}, \quad \forall 0<t \leq 1 .
$$

Proof of Lemma 2.1. The bound (2.6) is a well-known result; cf. [1]:

$$
\frac{1}{C_{1}} \min \left\{t^{-d / \alpha}, \frac{t}{|x|^{d+\alpha}}\right\} \leq p(t, x) \leq C_{1} \min \left\{t^{-d / \alpha}, \frac{t}{|x|^{d+\alpha}}\right\},
$$

which is clearly equivalent to (2.6). By (2.2) we have

$$
F_{\epsilon}(t, x)=p(t) * w(t)=\int_{\mathbb{R}^{d}} p(t, x-y) w(t, y) d y
$$

where

$$
\begin{aligned}
w(t, y) & =\epsilon t \int_{\mathbb{R}^{d}} \frac{e^{-\epsilon t \phi_{1}(\xi)}-1}{\epsilon t} e^{2 \pi i \xi \cdot y} d \xi \\
& =: \epsilon t w_{1}(t, y) .
\end{aligned}
$$

Consider for any $0<\theta \leq 1$, the function

$$
h_{\theta}(y)=\int_{\mathbb{R}^{d}} \frac{1-e^{-\theta \phi_{1}(\xi)}}{\theta} e^{2 \pi i \xi \cdot y} d \xi .
$$

For any $y=\left(y_{1}, \cdots, y_{d}\right)$, we have (note that $\phi_{1}(\xi)$ vanishes for $|\xi| \geq \frac{1}{3}$ and there is no problem with integration by parts)

$$
\begin{aligned}
2 \pi i y_{k} h_{\theta}(y) & =\int_{\mathbb{R}^{d}} \frac{1-e^{-\theta \phi_{1}(\xi)}}{\theta} \partial_{\xi_{k}} e^{2 \pi i \xi \cdot y} d \xi \\
& =-\int_{\mathbb{R}^{d}} e^{-\theta \phi_{1}(\xi)} \cdot \partial_{\xi_{k}} \phi_{1}(\xi) \cdot e^{2 \pi i \xi \cdot y} d \xi .
\end{aligned}
$$

From this and repeated integration by parts, it is not difficult to check that

$$
\max _{0<\theta \leq 1}\left|h_{\theta}(y)\right| \leq C(d, \alpha) \cdot \frac{1}{\left(1+|y|^{2}\right)^{d+10}}, \quad \forall y \in \mathbb{R}^{d} .
$$

Therefore for any $0<\epsilon t \leq 1$, we have

$$
\left|w_{1}(t, y)\right| \leq C(d, \alpha) \cdot \frac{1}{\left(1+|y|^{2}\right)^{d+10}}, \quad \forall y \in \mathbb{R}^{d} .
$$

Comparing this with (2.6), we get

$$
|w(t, y)| \leq C(d, \alpha) \cdot \epsilon \cdot p(t, y), \quad \forall y \in \mathbb{R}^{d}, \quad 0<t \leq 1 .
$$

Plugging it into (2.10) and using the fact that $p(t) * p(t)=p(2 t)$, we get

$$
\begin{aligned}
F_{\epsilon}(t, x) & \leq C(d, \alpha) \cdot \epsilon p(2 t, x), \\
& \leq \frac{1}{2} p(t, x), \quad \forall x \in \mathbb{R}^{d}, \quad 0<t \leq 1,
\end{aligned}
$$

where we used the fact $p(2 t, x) \leq$ const $\cdot p(t, x)$ and chose $\epsilon$ sufficiently small. Clearly $(2.7)$ follows. Finally (2.7) and (2.5) imply that $k_{\epsilon}(t, x)$ is positive everywhere. The bound (2.8) follows from Fourier transform and the fact that $\phi_{1}(0)=1$. 
Now we are ready to complete the

Proof of Theorem 1.1. By a scaling argument we only need to prove (1.4) for $N=1$. In view of the usual convolution property of the heat semigroup, i.e.,

$$
e^{-t|\nabla|^{\alpha}}=\left(e^{-\frac{t}{m}|\nabla|^{\alpha}}\right)^{m}
$$

it suffices to prove (1.4) for $0<t \leq 1$. Now recall that

$$
\widehat{P_{1} f}(\xi)=\psi(\xi) \hat{f}(\xi)
$$

where $\psi$ is compactly supported in the annulus $\{\xi: 1 / 2 \leq|\xi| \leq 2\}$. In view of this localization property, we can smoothly redefine the kernel $e^{-t(2 \pi|\xi|)^{\alpha}}$ on the tail part $\left\{\xi:|\xi|<\frac{1}{2}\right\}$ such that

$$
e^{-t(2 \pi|\xi|)^{\alpha}} \widehat{P_{1} f}(\xi)=e^{-t\left((2 \pi|\xi|)^{\alpha}+\epsilon \phi_{1}(\xi)\right)} \widehat{P_{1} f}(\xi), \quad \forall \xi \in \mathbb{R}^{d}
$$

where we choose $\phi_{1}(\xi)$ as in (2.1). Now denote $\phi_{\epsilon}(\xi)=(2 \pi|\xi|)^{\alpha}+\epsilon \phi_{1}(\xi)$.

Therefore, we only need to prove for $\epsilon>0$ sufficiently small,

$$
\left\|\left(\mathcal{F}^{-1}\left(e^{-t \phi_{\epsilon}}\right)\right) * P_{1} f\right\|_{q} \leq e^{-\epsilon t}\left\|P_{1} f\right\|_{q}, \quad \forall 0<t \leq 1
$$

But this follows easily from Lemma 2.1 and Young's inequality.

To prove Corollary 1.6, we need the following simple lemma.

Lemma 2.2. Let $0<\alpha \leq 2$. Let $f \in L_{\text {loc }}^{1}\left(\mathbb{R}^{d}\right)$. Then there is a constant $C_{\alpha, d}>0$ depending only on $(\alpha, d)$, such that

$$
\sup _{0<t<\infty}\left|\left(e^{-t|\nabla|^{\alpha}} f\right)(t, x)\right| \leq C_{\alpha, d}(M f)(x),
$$

where $M f$ is the Hardy-Littlewood maximal function defined as

$$
(M f)(x)=\sup _{B \ni x} \frac{1}{|B|} \int_{B}|f| .
$$

Proof of Lemma 2.2. Denote $p(y)=\mathcal{F}^{-1}\left(e^{-(2 \pi|\xi|)^{\alpha}}\right)$. By $(2.6)$ we have for some constant $C_{1}>0$,

$$
p(y) \leq C_{1} \cdot(1+|y|)^{-(d+\alpha)}
$$


Therefore for $0<t<\infty$,

$$
\begin{aligned}
& \left|\left(e^{-t|\nabla|^{\alpha}} f\right)(t, x)\right| \\
& \quad \leq \int_{\mathbb{R}^{d}} p(y)\left|f\left(x+t^{\frac{1}{\alpha}} y\right)\right| d y \\
& \quad \leq \int_{|y| \leq 1} p(y)\left|f\left(x+t^{\frac{1}{\alpha}} y\right)\right| d y+\sum_{k=1}^{\infty} \int_{2^{k-1} \leq|y| \leq 2^{k}} p(y)\left|f\left(x+t^{\frac{1}{\alpha}} y\right)\right| d y \\
& \quad \leq C_{1} \int_{|y| \leq 1}\left|f\left(x+t^{\frac{1}{\alpha}} y\right)\right| d y+C_{1} \sum_{k=1}^{\infty} 2^{-(k-1)(d+\alpha)} \int_{|y| \leq 2^{k}}\left|f\left(x+t^{\frac{1}{\alpha}} y\right)\right| d y \\
& \quad \leq C_{1} 2^{d+\alpha} \sum_{k \geq 0} 2^{-k \alpha} \sup _{k^{\prime} \geq 0} \int_{|y| \leq 1}\left|f\left(x+2^{k^{\prime}} t^{\frac{1}{\alpha}} y\right)\right| d y \\
& \quad \leq C_{d, \alpha}(M f)(x) .
\end{aligned}
$$

Proof of Corollary 1.6. Define

$$
F(t)=\int_{\mathbb{R}^{d}}\left|e^{-t|\nabla|^{\alpha}} P_{N} f\right|^{q} d x .
$$

Note that for each $t \geq 0$,

$$
\partial_{t}\left(e^{-t|\nabla|^{\alpha}} P_{N} f\right)=-e^{-t|\nabla|^{\alpha}}|\nabla|^{\alpha} P_{N} f .
$$

Since $1<q<\infty$, we have for any smooth function $b(t, x)$,

$$
\partial_{t}\left(|b(t, x)|^{q}\right)=-q \cdot\left(\partial_{t} b\right)(t, x)|b(t, x)|^{q-2} b(t, x) .
$$

Setting $b(t, x)=\left(e^{-t|\nabla|^{\alpha}} P_{N} f\right)(t, x)$, we get

$$
\partial_{t}\left(\left|\left(e^{-t|\nabla|^{\alpha}} P_{N} f\right)(t, x)\right|^{q}\right)
$$

$$
=-q \cdot\left(e^{-t|\nabla|^{\alpha}}|\nabla|^{\alpha} P_{N} f\right)(t, x) \cdot\left|\left(e^{-t|\nabla|^{\alpha}} P_{N} f\right)(t, x)\right|^{q-2} \cdot\left(e^{-t|\nabla|^{\alpha}} P_{N} f\right)(t, x) .
$$

By Lemma 2.2 and (2.13), we have

$$
\begin{aligned}
& \sup _{t \geq 0}\left|\left(\partial_{t}\left(\left|e^{-t|\nabla|^{\alpha}} P_{N} f\right|^{q}\right)\right)(t, x)\right| \\
& \quad \leq q M\left(|\nabla|^{\alpha} P_{N} f\right)(x) \cdot\left(M\left(P_{N} f\right)(x)\right)^{q-1} \\
& \quad \leq\left(M\left(|\nabla|^{\alpha} P_{N} f\right)(x)\right)^{q}+(q-1)\left(M\left(P_{N} f\right)(x)\right)^{q} \\
& \quad=: H(x) .
\end{aligned}
$$

Since $1<q<\infty$ and $f \in L_{x}^{q}\left(\mathbb{R}^{d}\right)$, it is easy to check that $H \in L_{x}^{1}\left(\mathbb{R}^{d}\right)$.

Now denote $b(t, x)=\partial_{t}\left(\left|e^{-t|\nabla|^{\alpha}} P_{N} f\right|^{q}\right)(t, x)$. Observe that by $(2.13)$, it is easy to check that $b$ is continuous in $(t, x)$ and consequently

$$
\lim _{\delta \downarrow 0} \frac{\int_{0}^{\delta} b(s, x) d s}{\delta}=-q\left(|\nabla|^{\alpha} P_{N} f\right)(x)\left|P_{N} f(x)\right|^{q-2} P_{N} f(x), \quad \forall x \in \mathbb{R}^{d} .
$$

Now let $0<h<1$ and write

$$
\frac{F(h)-F(0)}{h}=\int_{\mathbb{R}^{d}} \frac{\int_{0}^{h} b(s, x) d s}{h} d x .
$$


By (2.14), we have

$$
\sup _{0<h<1}\left|\frac{\int_{0}^{h} b(s, x) d s}{h}\right| \leq H(x) .
$$

By (2.15)-(2.16) and the Lebesgue Dominated Convergence Theorem, we obtain that $F$ is right-differentiable at $t=0$ and

$$
F^{\prime}(0+)=-q \int_{\mathbb{R}^{d}}\left(|\nabla|{ }^{\alpha} P_{N} f\right)(x)\left|P_{N} f(x)\right|^{q-2} P_{N} f(x) d x .
$$

In particular, we have

$$
\text { LHS of }(1.12)=-\frac{1}{q} F^{\prime}(0+) \text {. }
$$

On the other hand by using Theorem 1.1, we have for any $t>0$,

$$
\frac{F(0)-F(t)}{t} \geq \frac{1-e^{-c t q N^{\alpha}}}{t}\left\|P_{N} f\right\|_{q}^{q}
$$

Taking the limit $t \rightarrow 0$ immediately give us

$$
-F^{\prime}(0) \geq c q N^{\alpha}\left\|P_{N} f\right\|_{q}^{q}
$$

By (2.17) this gives us (1.12).

For the periodic case we recall the following standard Poisson summation formula.

Lemma 2.3 (Poisson summation formula). Let $f$ be a Schwartz function on $\mathbb{R}^{d}$ and denote by $\hat{f}$ its Fourier transform on $\mathbb{R}^{d}$. Then

$$
\sum_{n \in \mathbb{Z}^{d}} f(x+n)=\sum_{n \in \mathbb{Z}^{d}} \hat{f}(n) e^{2 \pi i n \cdot x}, \quad \forall x \in \mathbb{R}^{d} .
$$

As is well known, the Poisson summation formula holds for general continuous functions of moderate decrease including the heat kernels

$$
k_{\alpha}(t, \cdot)=\mathcal{F}^{-1}\left(e^{-t(2 \pi|\xi|)^{\alpha}}\right), \quad 0<\alpha \leq 2, t>0 .
$$

Denote the periodic kernel

$$
k_{\alpha}^{\mathrm{per}}(t, x)=\sum_{n \in \mathbb{Z}^{d}} e^{-t(2 \pi|n|)^{\alpha}} e^{2 \pi i n \cdot x} .
$$

The next lemma gives a lower bound on $k_{\alpha}^{\text {per }}(t, x)$. It is amusing that later this lower bound is used to prove an upper bound of the heat kernel.

Lemma 2.4 (Short time lower bound of the fractional heat kernel). Let the dimension $d \geq 1$ and let $0<\alpha<2$. There is a constant $c_{3}>0$ depending only on $(\alpha, d)$ such that

$$
k_{\alpha}^{p e r}(t, x) \geq c_{3} t, \quad \forall 0<t \leq 1, x \in \mathbb{T}^{d}
$$


Proof of Lemma 2.4. By Lemma 2.3 and (2.6), we have for $|x| \leq 1,0<t \leq 1$,

$$
\begin{aligned}
k_{\alpha}^{\mathrm{per}}(t, x) & =\sum_{n \in \mathbb{Z}^{d}} k_{\alpha}(t, x+n) \\
& \geq \sum_{n \in \mathbb{Z}^{d},|n|=3} k_{\alpha}(t, x+n) \\
& \geq c_{3} t
\end{aligned}
$$

where $c_{3}$ depends only on $(d, \alpha)$.

We now complete the

Proof of Theorem 1.9. We only need to show (1.14) since (1.15) follows from the same argument as in (1.8). Now assume that $0<\alpha<2$. By the convolution property we only need to prove the case $0<t \leq 1$. Since $\hat{f}(0)=0$ we may freely adjust the zeroth Fourier coefficient of the heat kernel. Doing so gives us

$$
e^{-t|\nabla|^{\alpha}} f=\tilde{k}(t, \cdot) * f,
$$

where

$$
\tilde{k}(t, x)=k_{\alpha}^{\mathrm{per}}(t, x)-c_{3} t
$$

By Lemma 2.4, we have

$$
\tilde{k}(t, x) \geq 0, \quad \forall 0<t \leq 1, x \in \mathbb{T}^{d} .
$$

Hence,

$$
\|\tilde{k}(t, \cdot)\|_{1}=1-c_{3} t \leq e^{-c_{1} t}, \quad \forall 0<t \leq 1 .
$$

Obviously (1.14) follows from the above bound and Young's inequality.

\section{Acknowledgments}

The author would like to thank Professor Ya.G. Sinai for informing him the book [10]. D. Li was supported in part by the NSF under agreement no. DMS-1128155. Any opinions, findings and conclusions or recommendations expressed in this material are those of the authors and do not necessarily reflect the views of the National Science Foundation. D. Li was also supported in part by an Nserc discovery grant. The author would like to thank the anonymous referee for very helpful comments and suggestions.

\section{References}

[1] R.M. Blumenthal and R.K. Getoor, Some theorems on stable processes, Trans. Amer. Math. Soc. 95 (1960), 263-273.

[2] D. Chamorro and P. Lemarié-Rieusset, Quasi-geostrophic equations, nonlinear Bernstein inequalities and $\alpha$-stable processes, Rev. Mat. Iberoam. 28(4) (2012), 1109-1122.

[3] J. Chemin Théorèmes d'unicité pour le système de Navier-Stokes tridimensionnel, [Uniqueness theorems for the three-dimensional Navier-Stokes system] J. Anal. Math. 77 (1999), 27-50.

[4] Q. Chen, C. Miao and Z. Zhang, A new Bernstein's inequality and the 2D dissipative quasigeostrophic equation, Commun. Math. Phys. 271(3) (2007), 821-838.

[5] R. Danchin, Poches de tourbillon visqueuses, J. Math. Pures Appl. 76(9), 609-647 (1997).

[6] R. Danchin, Local theory in critical spaces for compressible viscous and heat-conductive gases, Commun. Part. Differ. Equ. 26 (2001), (7-8), 1183-1233.

[7] R. Danchin, Erratum: "Local theory in critical spaces for compressible viscous and heatconductive gases" [Commun. Part. Differ. Equ. 26 (2001), (7-8), 1183-1233.] Commun. Part. Differ. Equ. 27(11-12) (2002), 2531-2532. 
[8] H. Dong and D. Li, On a generalized maximum principle for a transport-diffusion model with log-modulated fractional dissipation, arXiv:1209.3701.

[9] T. Hmidi, On a maximum principle and its application to the logarithmically critical Boussinesq system, Anal. PDE, 4(2) (2011), 247-284.

[10] S. Kuksin and A. Shirikyan, Mathematics of two-dimensional turbulence, Cambridge Tracts in Mathematics 194. Cambridge Unversity Press, 2012.

[11] F. Planchon, Sur une inégalité de type Poincaré, Notes aux Comptesrendus de l'Académie des Sciences de Paris 2000, 330, 21-23.

[12] J. Wu, Lower bounds for an integral involving fractional Laplacians and the generalized NavierStokes equations in Besov spaces, Commun. Math. Phys. 263 (2006), 803-831.

Department of Mathematics, University of British Columbia, Vancouver, BC V6T 1Z2, CANADA

E-mail address: mpdongli@gmail.com 
